Table $1 \mathrm{BMI}$ and metabolic parameters in obese children with IGT ofter a 6-month diet and lifestyle changes $(n 32)$

\begin{tabular}{|c|c|c|c|c|}
\hline & \multicolumn{2}{|c|}{ 1st examination } & \multicolumn{2}{|c|}{ 2nd examination } \\
\hline & Mean & SD & Mean & SD \\
\hline BMI $\left(\mathrm{kg} / \mathrm{m}^{2}\right)$ & $30 \cdot 4$ & $4 \cdot 9$ & $29 \cdot 0$ & $4 \cdot 4^{\star \star}$ \\
\hline Plasma glucose $120^{\prime}(\mathrm{mmol} / \mathrm{l})$ & $8 \cdot 6$ & $0 \cdot 7$ & $7 \cdot 0$ & $1 \cdot 2^{*}$ \\
\hline Plasma insulin $0^{\prime}(\mathrm{ml} \mathrm{U} / \mathrm{ml})$ & $29 \cdot 1$ & $9 \cdot 2$ & $18 \cdot 8$ & $8 \cdot 0^{*}$ \\
\hline Plasma insulin $120^{\prime}(\mathrm{ml} \mathrm{U} / \mathrm{ml})$ & $168 \cdot 7$ & $92 \cdot 9$ & $117 \cdot 4$ & $85 \cdot 9^{\star}$ \\
\hline HOMA & $6 \cdot 7$ & $3 \cdot 7$ & $4 \cdot 9$ & $3 \cdot 3^{\star}$ \\
\hline
\end{tabular}

IGT, impaired glucose tolerance; HOMA, homeostasis model assessment. ${ }^{\star} P<0.001,{ }^{\star \star} P<0.05$.

IGT or T2DM in clinically healthy obese children can - at least temporarily - be managed with dietary and lifestyle interventions, resulting in the improvement of the metabolic status of these children. It is known that many of the metabolic, cardiovascular and oncologic consequences of obesity are likely influenced through insulin resistance and production of inflammatory adipokines. Although diagnostic strategies are almost clear, and the majority of the changes of hormones and adipokines measured in obese children are reversible after weight loss, however treatment remains difficult, so prevention should be started very early in life. The current knowledge of adipokines, different hormones and the production of proinflammatory factors involved in the pathogenesis of insulin resistance will be also discussed.

\title{
Vitamin D deficiency is highly prevalent in obese children and adolescents and associated with decreased insulin sensitivity
}

\author{
G Massa ${ }^{1}$, A Vanoppen ${ }^{1}$, W Achten ${ }^{1}$ and A Mewis ${ }^{2}$ \\ 'Department of Paediatrics, Jessa Hospital, Hasselt, Belgium: ${ }^{2}$ Clinical Laboratory, Jessa Hospital, Hasselt, Belgium
}

Introduction: Low 25-hydroxyvitamin D (25(OH)D) is one of the endocrine derangements in obesity. We evaluated the prevalence of vitamin D deficiency $(<15 \mathrm{ng} / \mathrm{ml})$ in obese children and adolescents and studied the relationship with BMI, ethnicity, season and insulin sensitivity.

Method: Fasting serum 25(OH)D, glucose and insulin levels and the quantitative insulin sensitivity check index (QUICKI) were determined in ninety-one subjects aged $13 \cdot 2$ (SD 1.9) years (sixty-eight autochtones, twenty-three allochtones; 56\% female; BMI-SDS 2.7 (SD 0.5) during fall/winter (F/W; $n$ 56) and spring/summer (S/S; $n$ 35).

Results: Vitamin D deficiency was present in $57 \%$ of the cohort. It was more prevalent in $\mathrm{F} / \mathrm{W}$ than $\mathrm{S} / \mathrm{S}(68 \% v$. 40\%, $P<0 \cdot 02)$. Patients with vitamin $\mathrm{D}$ deficiency had higher fasting insulin levels (25 (SD 14) qU/ml v. 19 (SD 10) qU/ml; $P<0 \cdot 02)$ and lower QUICKI $(0.308$ (sD 0.026$) v .0 \cdot 320$
(SD 0.028); $P<0 \cdot 05)$, but comparable BMI (2.8 (SD 0.5) SDS v. $2 \cdot 7(\mathrm{SD} 0 \cdot 5) \mathrm{SDS})$. Serum $25(\mathrm{OH}) \mathrm{D}$ levels were inversely related to fasting insulin levels $(r=-0 \cdot 29 ; P<0 \cdot 01)$ and positively to QUICKI $(r=+0 \cdot 31 ; P<0 \cdot 005)$, but not to BMI-SDS $(r=-0 \cdot 16)$. Multiple regression analysis revealed that serum $25(\mathrm{OH}) \mathrm{D}$ levels were related to season $(T=+3 \cdot 6 ; P=0 \cdot 001)$, ethnicity $(T=-2 \cdot 9 ; P=0 \cdot 004)$ and QUICKI $(T=+2 \cdot 3 ; P=0 \cdot 022)$, but not to BMI-SDS.

Conclusions: Vitamin D deficiency is highly prevalent in obese children and adolescents; vitamin D status is influenced by season and ethnicity but not by BMI. Furthermore, serum 25(OH)D levels were positively related to insulin sensitivity suggesting that obese children and adolescents with hypovitaminosis D are at increased risk of developing impaired glucose metabolism independent of BMI.

\section{Correlation of ghrelin and obestatin levels with tryptophan degradation in obese children}

D Zaknun ${ }^{1}$, D Weghuber ${ }^{2,3}$, V Krösslhuber ${ }^{2}$ and D Fuchs ${ }^{4}$

${ }^{1}$ Department of Paediatrics, Medical University of Vienna, Vienna, Austria: ${ }^{2}$ Department of Paediatrics, Paracelsus Private Medical School Salzburg, Salzburg, Austria: ${ }^{3}$ Obesity Academy Austria, Austria: ${ }^{4}$ Division of Biological Chemistry, Biocenter, Innsbruck Medical University, Innsbruck, Austria 